\title{
La modernité dans l'historiographie du Soudan
}

Usages convenus d'un concept nébuleux?

Scrutinizing modernity through the historiography of Sudan: conventional uses

of a nebulous concept?

\section{Iris Seri-Hersch}

\section{OpenEdition \\ Journals}

Édition électronique

URL : http://journals.openedition.org/etudesafricaines/17197

DOI : 10.4000/etudesafricaines. 17197

ISSN : $1777-5353$

\section{Éditeur}

Éditions de l'EHESS

\section{Édition imprimée}

Date de publication : 5 octobre 2012

Pagination : 905-935

ISBN : 978-2-7132-2350-1

ISSN : 0008-0055

\section{Référence électronique}

Iris Seri-Hersch, «La modernité dans I'historiographie du Soudan », Cahiers d'études africaines [En ligne], 208 | 2012, mis en ligne le 13 octobre 2014, consulté le 02 mai 2019. URL : http:// journals.openedition.org/etudesafricaines/17197; DOI : 10.4000/etudesafricaines. 17197

Ce document a été généré automatiquement le 2 mai 2019.

(c) Cahiers d'Études africaines 


\title{
La modernité dans l'historiographie du Soudan
}

\author{
Usages convenus d'un concept nébuleux? \\ Scrutinizing modernity through the historiography of Sudan: conventional uses \\ of a nebulous concept?
}

Iris Seri-Hersch

1 Formulée par saint Augustin il y a seize siècles, cette réflexion garde toute sa pertinence à l'ère de la révolution informatique. Le temps, symbolisé et matérialisé par des outils de plus en plus sophistiqués, demeure une énigme pour l'esprit humain. En butte contre son caractère insaisissable, les sociétés humaines ont élaboré des dispositifs visuels et cognitifs permettant d'imaginer l'expérience humaine sur la durée, la rendant plus accessible. Le concept de modernité, au sujet duquel je propose une réflexion critique dans le contexte de l'historiographie soudanaise, soulève le problème crucial du rapport entre le présent et le passé, deux notions certes volatiles mais centrales dans l'expérience individuelle et la vie en société.

2 Cette recherche ${ }^{1}$ se fonde sur une distinction heuristique fondamentale, rappelée récemment par un historien spécialiste de l'Afrique et des études coloniales : celle entre catégories normatives et catégories analytiques. Les premières sont employées par les acteurs historiques, les secondes par les chercheurs en sciences sociales. Les catégories normatives sont donc des catégories de pratique, caractéristiques de la vie quotidienne. Par contraste, les catégories analytiques comptent parmi les outils de la recherche scientifique (Cooper $2005: 62$ ).

3 Procédant d'une démarche qui se veut à la fois théorique et empirique, j'appréhenderai la modernité en tant que catégorie d'analyse construite par des historiens du Soudan, dont la production académique constitue elle-même l'objet de ma recherche. Il s'agit donc de déconstruire les usages savants d'un concept qui, malgré - ou grâce à - son éclatement sémantique, n'en finit pas de nourrir des débats animés dans le monde universitaire, en particulier dans le domaine des études (post-) coloniales². 
4 Le corpus de sources sélectionné pour cette étude comprend des articles et ouvrages d'historiens britanniques, soudanais, français, néerlandais, américains, japonais et israéliens portant sur une variété d'aspects du Soudan contemporain, publiés entre 1959 et 2008. Quel(s) sens les soudanistes attribuent-ils à la modernité et comment emploientils ce concept dans leurs recherches?

5 L'emploi même du terme "modernité » sous-tend une certaine vision de l'expérience humaine, divisée en périodes distinctes par les historiens afin de mieux pouvoir la cerner. J'esquisserai tout d'abord la façon dont la modernité a été pensée en France et en GrandeBretagne durant la seconde moitié du $\mathrm{xx}^{\mathrm{e}}$ siècle. Ces conceptions européennes sont pertinentes pour notre réflexion dans la mesure où elles ont fréquemment été appliquées à des régions non européennes, dont le Soudan. Ensuite, l'analyse des usages académiques de la modernité dans l'historiographie du Soudan sera présentée à travers, d'une part, des travaux historiques de large amplitude (dont les sujets sont généraux et couvrent des périodes de plus d'un demi-siècle); d'autre part, à travers une sélection d'études plus pointues qui traitent explicitement ou implicitement de la question de la modernité. Nous nous poserons la question de savoir si la grande diversité des approches et des objets caractérisant ces études correspond à des acceptions du concept de modernité également variées.

\section{Conceptions européennes de la modernité : maintenant ou époque révolue?}

6 Le terme « moderne » est issu du grec « modos » qui signifie aujourd'hui. Le sens premier de moderne se réfère à ce qui caractérise le présent. La modernité est donc mouvante, elle correspond à «l'époque » d'un groupe humain telle que vécue par lui et distinguée par lui d'une époque antérieure. Cependant, la modernité peut aussi renvoyer à une période historique déterminée, dont les bornes à l'intérieur du flux incessant des événements varient en fonction des écoles historiques et des régions du monde examinées. Ces deux façons différentes de conceptualiser la modernité ont coexisté en Europe au cours des derniers siècles. Les diverses sciences et disciplines de la connaissance humaine ont contribué à les distinguer, en imposant chacune dans son champ un ou plusieurs usages de la notion.

7 Dans le domaine littéraire, la querelle des Anciens et des Modernes (dans l'Italie et la France des $\mathrm{XVII}^{\mathrm{e}}$-XVIII ${ }^{\mathrm{e}}$ siècles) met en relief le fait d'être moderne comme une attitude qui prône l'innovation créatrice en même temps qu'elle rejette certaines traditions littéraires et règles d'écriture associées au passé, notamment à l'Antiquité. Cependant, la paternité du terme français "modernité » est attribuée au poète romantique François-René de Chateaubriand (1768-1848) qui l'utilise pour contraster la banalité de la vie "moderne » avec l'éternelle sublimité de la nature et la grandeur d'un passé médiéval légendaire (Călinescu 1987: 43). Un peu plus tard, Charles Baudelaire fixe un certain usage esthétique de la "modernité » en la décrivant comme «le transitoire, le fugitif, le contingent, la moitié de l'art, dont l'autre moitié est l'éternel et l'immuable » (Ruff 1968 : 74).

8 La modernité telle qu'elle a été conceptualisée par des historiens européens durant la seconde moitié $\mathrm{du} \mathrm{xx}^{\mathrm{e}}$ siècle privilégie la représentation d'une période historique déterminée, bien que son emplacement sur le flux des événements fasse débat. Il y a tout 
d'abord des divergences d'ordre linguistique entre les historiens français et leurs homologues anglo-saxons. Alors que les premiers ont pris l'habitude de désigner par l'appellation «Temps modernes » la période qui s'étend de la fin du Moyen-Âge à la Révolution française, les seconds prolongent volontiers les « Modern Times » jusqu'à nos jours (divisant cette durée de cinq siècles en « Early Modern Times » et " Modern Times », les Révolutions américaine et française marquant la charnière entre les deux) ${ }^{3}$. L'expression anglophone "modern history» ne se traduira généralement pas par " histoire moderne", mais bien plutôt par " histoire contemporaine", correspondant ainsi aux $\mathrm{XIX}^{\mathrm{e}}$ et $\mathrm{XX}^{\mathrm{e}}$ siècles. En dépit de ces différences formelles, la majorité des historiens européens - qu'ils soient français, anglo-saxons, allemands ou autres semblent s'accorder sur les composantes essentielles de ce qu'ils désignent par le terme de «modernité ». Leurs divergences ont trait au poids relatif des différents phénomènes identifiés, ainsi qu'aux liens entre eux. Quels sont les ingrédients de cette modernité ?

Selon l'historien et politologue Pascal Ory, les Temps modernes sont pour l'Europe le moment d'une rupture significative par rapport au passé, marquée par deux changements cruciaux : l'entrée de l'Occident dans l'ère de la relativité et l'instauration de l'État (Ory 1987 : 29). La relativité est le fruit de plusieurs développements parallèles. Premièrement, les découvertes géographiques européennes $\mathrm{du} \mathrm{xv}^{\mathrm{e}}$ siècle et les empires intercontinentaux qui leur succèdent produisent un nouvel espace/temps dans l'esprit des Européens. Deuxièmement, l'essor du mouvement scientifique autour de figures comme Copernic, Galilée et Newton contribue à ébranler les convictions médiévales voire antiques. Enfin, la Réforme protestante individualise le sujet en accordant une importance primordiale au dialogue direct avec Dieu, «libérant» en quelque sorte le croyant de la médiation imposée par les institutions cléricales ${ }^{4}$. Au niveau politique, les Temps modernes marquent l'apogée du système absolutiste en même temps que l'élaboration de la notion d'État de droit par des penseurs novateurs tels que Thomas Hobbes (ibid. : 30).

10 Considérant la modernité dans une perspective plus large, le politologue René Rémond (1974 : 198) met en évidence les rôles respectifs du mouvement des idées philosophiques et de la Révolution française dans la rupture avec les modes de pensée et d'organisation sociale prévalents au Moyen-Âge. Le mouvement des idées du XVIII ${ }^{\mathrm{e}}$ siècle - non sans prétention surnommé «Lumières" - annonce la modernité au sens où les philosophes affirment la capacité de la raison à régler tous les aspects de l'existence humaine. Cette position porte en germe la laïcisation de l'État ainsi que la sécularisation de la société. Quant à la Révolution française, elle joue un rôle crucial dans l'application juridique des principes revendiqués par le mouvement philosophique, même si cette réalisation ne se fait qu'un siècle plus tard. À titre d'exemple, rappelons que dans le contexte français, les Églises et l'État ne divorcent officiellement qu'en 1905.

11 Encore plus que Rémond, l'historien Jonathan Israel (2001: 23) insiste sur le rôle prépondérant des Lumières dans la "naissance » de la modernité. Il voit dans les phénomènes de la Renaissance et de la Réforme de simples ajustements d'une société qui continue à fonctionner selon des concepts théologiques. En outre, ces changements sont limités à l'Europe occidentale et centrale. Par contraste, les Lumières sont l'expression d'un phénomène d'une envergure considérablement plus vaste : elles représentent «l'un des bouleversements les plus importants de l'histoire de l'humanité » (ibid.). En effet, ce sont elles qui mettent en branle les processus de sécularisation de la société et de rationalisation de la politique. Dans son enthousiasme pour le mouvement philosophique, 
Israel n'hésite pas à lui reconnaître l'éradication de nombreux « maux » qui disparaissent en fait bien plus tard, sinon qui n'ont pas encore disparu. Ainsi, il déclare qu'«[anéantissant] toute légitimation de la monarchie, de l'aristocratie, de la subordination des femmes aux hommes, de l'autorité ecclésiastique et de l'esclavage " (ibid.), les Lumières substituent à ces fondements les principes d'universalité, d'égalité et de démocratie.

12 L'une des idées maîtresses de la philosophie des Lumières, étroitement associée à la modernité dans l'esprit de nombre d'intellectuels, est celle de progrès. À l'aube du XIX siècle, Condorcet (1793) propose une synthèse de cette idée dans son Esquisse d'un tableau historique des progrès de l'esprit humain. Le progrès, c'est «le triomphe progressif de la raison sur les croyances, de la vérité sur l'obscurité, de la science sur la religion, de la liberté sur le despotisme» (Bouretz 1987 : 369). Dans cette perspective, les penseurs des Lumières fixent un idéal de la connaissance par la science et un idéal d'organisation de la société par la volonté. Le xix ${ }^{e}$ siècle voit l'idée de progrès s'envelopper d'une myriade d'ambiguïtés. En effet, la question de savoir si le progrès des connaissances et des techniques permet un modèle de progrès social trouvera une variété de réponses à travers des théories telles que le positivisme d'Auguste Comte (ordre et progrès), la philosophie hégélienne de l'histoire (la Raison comme moteur de l'histoire et l'État comme incarnation de cette Raison), et le marxisme (la lutte des classes comme moteur de l'Histoire) (ibid.: 371-373). En se popularisant, l'idée de progrès semble perdre son sens, puisque celui-ci se dilue dans diverses théories. Mentionnant plusieurs types de progrès (progrès-liberté, progrès-pouvoir, progrès-autonomie, progrès-ordre), Pierre Bouretz met en évidence l'ambiguïté de cette notion, caractéristique de la modernité :

«L'idée de progrès n'a peut-être pas tant un sens en elle-même que rapportée à des pensées qui l'articulent à d'autres concepts, qui la polarisent dans différentes directions en fonction de visions plus larges des finalités de la société, de son organisation, de la régulation de ses conflits. »(ibid. : 375-376)

$\mathrm{Au} \mathrm{Xx} \mathrm{e}^{\mathrm{e}}$ siècle, nombreux sont les penseurs qui remettent en question la pertinence même du concept de progrès. Parmi eux, Karl Popper $(1956: 153)$ critique l'usage téléologique du progrès identifié à l'historicisme. Toutefois, il ne rejette pas l'idée d'un progrès scientifique résultant de la libre concurrence de la pensée. Raymond Aron (1969: 282) reconnait le progrès scientifique et technique comme l'un des pans de la modernité, potentiellement commun à toute l'humanité. Néanmoins, la modernité, c'est aussi le pluralisme des ordres sociaux, des créations artistiques et des croyances religieuses qui caractérisent les groupes humains.

Parmi une multitude de "progrès " possibles, il en est un qui est devenu profondément associé à la modernité, ceci au-delà des différences de périodisation mentionnées plus haut. Il s'agit du progrès technique, et plus particulièrement des développements et des inventions que l'on rassemble sous le label de « Révolution industrielle ». Cette révolution et les nouvelles formes de stratification sociale qu'elle engendre ne se produisent pas avant la fin du XVIII ${ }^{e}$ siècle pour la Grande-Bretagne et le premier tiers du siècle suivant pour la France. Ainsi, ces bouleversements sont postérieurs au cadre des Temps modernes tel qu'il a été défini par l'historiographie française (de la fin du Moyen-Âge jusqu'à la Révolution française). Cependant, il est remarquable que des intellectuels aussi bien français, allemands qu'anglo-saxons établissent une quasi-synonymie entre les expressions «sociétés modernes » et «sociétés industrialisées » ${ }^{5}$. On peut apprécier la 
force de cette idée en observant la banalité qu'il y a, jusqu'à aujourd'hui, à interchanger les termes « moderne » et « industrialisé » dans nombre d'énoncés relatifs aux sociétés.

Enfin, la modernité est généralement associée avec la production d'idéologies nouvelles, dénommées par des -ismes. Ainsi en est-il du libéralisme, du socialisme et du nationalisme. Ce dernier a fait l'objet d'un nombre impressionnant d'études durant le dernier quart $\mathrm{du} \mathrm{xx}^{\mathrm{e}}$ siècle ${ }^{6}$, qui se sont employées à démontrer son caractère éminemment moderne malgré un discours qui s'enracine dans l'histoire. L'étonnante longévité des idéologies nationalistes, ainsi que leur pouvoir mobilisateur extrêmement fort au $\mathrm{xx}^{\mathrm{e}}$ siècle, expliquent l'intérêt académique marqué dont elles ont joui ces dernières décennies.

Benedict Anderson (2002: 19), de par sa célèbre définition de la nation en tant que "communauté politique imaginaire et imaginée comme intrinsèquement limitée et souveraine ", souligne le caractère moderne du phénomène national. Il ne peut en être autrement, puisque c'est la conjonction du développement du capitalisme marchand et de l'invention de l'imprimerie qui permet l'émergence de «communautés imaginées ». La possibilité de se rattacher à autrui en des termes radicalement nouveaux naît de ces changements qui interviennent à partir du début du XVI e siècle (ibid. : 47). Poursuivant la même approche, Ernest Gellner affirme que le nationalisme est l'une des conséquences de l'organisation sociale industrielle et de l'homogénéisation linguistique, phénomènes typiques de la modernité. Il propose une typologie des nationalismes en combinant les paramètres qui, selon lui, sont essentiels dans la constitution des sociétés modernes : la forme du pouvoir politique, l'accès à l'éducation et le monoculturalisme ou multiculturalisme d'une société donnée (Gellner 1999 : 129-156) ${ }^{7}$.

Se terminant également en -isme, mais système plutôt qu'idéologie, le capitalisme est un marqueur central de la modernité dans la littérature académique européenne. L'essor du capitalisme en tant que régime basé sur la propriété privée des moyens de productions, l'accumulation constante de capital, la recherche du profit et la liberté des échanges économiques est étroitement associé à l'avènement de l'âge moderne en Europe ${ }^{8}$.

Associée à une période de l'histoire européenne, la modernité est pensée comme un ensemble de phénomènes et de processus historiques dont le poids est variable, mais qui peuvent se résumer ainsi : un mode de pensée relativiste, l'instauration de l'État et sa laïcisation, la sécularisation de la société, la rationalisation de la politique, le progrès scientifique, l'industrialisation, la croissance du système économique capitaliste, la production d'idéologies proposant de nouveaux modèles d'ordre social. La modernité s'apparente donc plus à une notion nébuleuse qu'à un outil analytique précis. Examinons à présent quelles formes épouse la modernité lorsqu'elle est déployée dans le champ de l'histoire soudanaise. Les conceptions esquissées ci-dessus sont-elles schématiquement transposées en amont de la vallée du Nil ? Les soudanistes ont-ils réussi à faire de la modernité une catégorie d'analyse féconde pour leurs recherches?

\section{Le Soudan et la modernité : approches globales}

Un coup d'œil aux études traitant de la modernité au Soudan suffit à faire apparaître un glissement lexical notable, qui implique un glissement sémantique important. Plutôt que d'employer le terme "modernité », la plupart des historiens emploient celui de «modernisation ». Les deux termes peuvent être distingués ainsi : alors que la modernité 
renvoie à une certaine époque, telle qu'elle est vécue par l'Europe, la modernisation se réfère au processus qui transforme une entité en entité moderne, lui permettant « d'accéder » à la modernité. La société qui vit la modernisation - soudanaise dans notre cas - semble avoir besoin d'un processus actif pour devenir "moderne»; elle ne le devient pas par elle-même. L'usage même du terme "modernisation» se fait donc inévitablement par rapport à une modernité qui sert de référence. La question est de savoir si cette modernité est nécessairement européenne.

La majorité des travaux de portée générale sur le Soudan reprend les théories de la modernisation élaborées durant les années 1950 et 1960. Quelques-uns se calquent sur les modèles des théories de la dépendance formulées au cours des années 1960 et 1970 en réaction aux théories de la modernisation. Parmi les travaux analysés ici, aucune étude de vaste amplitude n'échappe fondamentalement à l'une de ces deux tendances.

\section{La modernisation comme processus d'inclusion dans l'ordre occidental}

21 Théories socio-économiques, les théories de la modernisation mettent en évidence le rôle positif du monde « développé » (un qualificatif qui dans bien des cas s'est substitué à celui de « moderne ») dans la modernisation de pays « sous-développés » (pays qui, à l'époque contemporaine, sont perçus comme inférieurs sur les plans technique, économique, mais également politique et culturel). Dans la pratique, le monde non occidental doit pouvoir bénéficier de la guidance de l'Occident à travers l'internalisation de valeurs modernes occidentales. Les théories de la modernisation se fondent sur des principes imprégnés d'évolutionnisme social: premièrement, les sociétés passent inexorablement par des stades de développement unidirectionnels qui transforment les sociétés dites «traditionnelles » en sociétés dites «modernes "; deuxièmement, les pays occidentaux sont au plus haut niveau de développement par rapport au reste du monde. Celui-ci, constitué de nombreuses ex-colonies occidentales, est à un stade plus primitif de développement. Il a besoin d'une intervention extérieure pour « avancer » et atteindre finalement le même niveau que le monde occidental'.

La plupart des études globales examinées ici adhèrent, à quelques nuances près, au modèle prôné par les théories de la modernisation. Les processus de modernisation y sont donc identifiés à des processus d'européanisation ou d'occidentalisation. Nous analyserons ces travaux dans l'ordre chronologique de leur publication afin de pouvoir observer une évolution sémantique potentielle, au cours du temps, du concept de «modernisation » dans l'historiographie du Soudan.

Dans son ouvrage devenu classique pour l'étude du Soudan ottomano-égyptien (1820-1885), Richard Hill (1959) met en relief l'importance de l'héritage égyptien dans l'histoire contemporaine du Soudan ${ }^{10}$. Cet héritage se traduit d'un côté par un processus de modernisation - dont l'inspiration est largement européenne, de l'autre côté par une influence socio-culturelle spécifiquement égyptienne. La modernisation se produit dans les domaines suivants : l'agriculture (rendement plus élevé), la perception des impôts (efficacité accrue), les communications (développement du télégraphe, introduction de bateaux à vapeur, établissement d'un service postal) et le commerce (croissance des exportations de gomme arabique) (ibid. : 153-158). Ces innovations avant tout techniques sont accompagnées d'une présence européenne croissante au Soudan, en particulier dans la capitale Khartoum. Explorateurs, scientifiques, commerçants, missionnaires ou 
employés par le gouvernement égyptien, les Européens y introduisent graduellement des produits importés d'Europe, tels que de la nourriture en boîte de conserve, des médicaments ou un style architectural nouveau (ibid. : 160-161) ${ }^{11}$. Quant à l'héritage socioculturel égyptien, il se reflète dans plusieurs sphères. Certaines institutions administratives ottomano-égyptiennes ont été conservées ou légèrement modifiées sous le régime du Condominium anglo-égyptien (1899-1956), par exemple les titres, les fonctions, les frontières provinciales et le vocabulaire administratif. De profondes attaches religieuses ont continué à nouer le Soudan à l'Égypte (formation de Soudanais à al-Azhar, diffusion d'un sunnisme orthodoxe au Soudan). Enfin, la culture égyptienne a eu un impact significatif sur le Soudan (tourisme soudanais en Égypte et émigration de main-d'œuvre soudanaise vers Le Caire, popularité de la production littéraire et cinématographique égyptienne auprès des couches soudanaises instruites) (ibid.: 167-169). Sous la plume de Hill ${ }^{12}$, le règne ottomano-égyptien au Soudan marque le début de l'histoire contemporaine soudanaise. Cette modernité se caractérise donc par une double influence : celle des Européens, notamment dans le domaine des sciences et des techniques, et celle des Égyptiens, prégnante dans les sphères culturelle, religieuse et administrative. Le facteur extérieur (européen et égyptien) est ici extrêmement significatif : c'est lui qui, d'une certaine manière, a permis la modernisation du Soudan.

Deux ans après Hill, les historiens Peter Holt et Martin Daly (2000 : VIII) adoptent son approche en la modifiant quelque peu. Dans la préface de leur ouvrage commun, ils affirment que :

«Les premières mesures menant à la construction du Soudan moderne furent prises il y a quasiment un siècle et demi, lorsque les soldats de Muhammad Ali Pasha, vice-roi du sultan ottoman en Égypte, amenèrent sous le règne de leur maitre les cultivateurs, marchands et hommes de tribu musulmans de Nubie, de Sennar et du Kordofan $»^{13}$.

Les débuts de la modernité au Soudan semblent se préciser, remontant à la conquête ottomano-égyptienne de 1820-1821. L'unification politico-administrative du territoire représente le moteur primordial de cette modernité ${ }^{14}$. Les auteurs établissent ensuite une relation directe et linéaire entre l'expérience de la domination étrangère (ottomanoégyptienne puis anglo-égyptienne), l'unification politique du Soudan, le développement du nationalisme soudanais et l'émergence d'un État soudanais indépendant en 1956. Bien qu'ils reconnaissent la prééminence de trois facteurs dans l'histoire contemporaine du Soudan (la tradition « indigène $»^{15}$, l'influence égyptienne et l'influence britannique), Holt et Daly soulignent que le Soudan contemporain est politiquement, matériellement et culturellement l'héritier des régimes égyptiens et britanniques. Comme chez Hill, l'agent extérieur joue un rôle essentiel dans le processus qui fait «entrer» le Soudan dans la modernité. Cependant, celle-ci se caractérise plutôt par le couple impérialismenationalisme que par les progrès techniques importés d'Europe.

Les mêmes historiens ont plus tard proposé, chacun de son côté, une vision particulière du phénomène de la modernisation dans le contexte soudanais. L'apparent contraste entre les deux conceptions est tout à fait intéressant. En 1973, Holt définit ainsi la modernisation :

«Par "modernisation", j'entends l'introduction de méthodes d'organisation politique et économique et de techniques de production, de transport et de communication dérivées de celles employées dans les pays européens. Elles [ces méthodes et techniques] ont toutes modifié substantiellement la structure de la société traditionnelle antérieure. »(Holt $1973:$ 135) 
agit d'une forme d'européanisation qui intervient essentiellement sur les plans politique, économique et technique. Douze ans plus tard, Daly (1985 : 11) reprend la formule de son collègue en y apportant une modification qui, bien qu'elle puisse sembler à première vue salutaire, contribue à épaissir le brouillard sémantique entourant la notion de modernisation. En effet, il écrit :

«Dans cet ouvrage, aucun de nous [les contributeurs] n'emploie "modernisation" pour signifier "occidentalisation" ou la simple imitation d'une culture étrangère. Nous évitons la tornade du débat sociologique en acceptant largement la façon dont P. M. Holt utilise "modernisation" pour signifier [...] [voir citation précédente] (nous émettons une réserve : que les facteurs introduits n'ont pas nécessairement pour origine l'Europe.) Ainsi, la perception est celle d'un changement plutôt que d'un progrès. »

Cet énoncé soulève deux problèmes majeurs quant au sens même du concept de modernisation. D'un côté, la réserve émise entre parenthèses permet à l'auteur de se distancer des théories de la modernisation en dissociant celle-ci des processus d'européanisation. La modernisation renvoie à tout changement politique, économique ou technique affectant une société "traditionnelle». Ainsi nuancé et élargi, le sens du concept s'évapore dans des généralités. En effet, si la modernisation a le sens de n'importe quel changement (ni forcément lié à l'Europe, ni nécessairement perçu comme un progrès), à quoi sert cette notion? Elle devient vague au point d'en perdre son efficacité, son utilité, donc sa pertinence ${ }^{16}$.

D'un autre côté, Daly paraît épouser la perspective des théories de la modernisation - à son insu? - tout en la dénonçant. Lorsqu'il lie la réserve émise entre parenthèses à la phrase suivante par un connecteur logique (thus que j'ai traduit par «ainsi »), il crée l'impression que voici : puisque les facteurs introduits dans la société «traditionnelle » ne sont pas nécessairement d'origine européenne, la modernisation est synonyme de changement plutôt que de progrès. En d'autres termes, le progrès impliquerait l'introduction de facteurs d'origine européenne. C'est là l'un des principes fondamentaux des théories de la modernisation. En conséquence, bien que Daly (1985 : 11) déclare éviter la «tornade du débat sociologique », son énoncé, voulu simple et presque anodin, peut susciter un débat florissant sur le sens même du concept de « modernisation ».

Au début des années 1990, Gabriel Warburg (1992: 3) conçoit la modernité sur la base des éléments signalés jusqu'à présent (le règne ottomano-égyptien comme l'instaurateur d'un gouvernement centralisé, la modernisation des méthodes employées dans l'agriculture et le commerce, l'amélioration des communications, l'émergence du nationalisme soudanais) tout en y ajoutant des éléments nouveaux : l'émergence d'une classe moyenne soudanaise s'appuyant sur des biens fonciers et humains (esclaves) ainsi que sur ses liens avec l'élite "turque"; un processus d'industrialisation exemplifié par la culture cotonnière et l'usine de filature établies dans l'Est du Soudan (région de Kassala) à la fin des années 1860 ; l'européanisation de l'empire égyptien sous l'impact de l'idéologie laïque du khédive Ismaïl. Bien qu'il adopte globalement l'approche défendue par les théories de la modernisation, Warburg tâche de mettre en évidence des processus endogènes au Soudan, tels que l'apparition d'une nouvelle classe socio-économique et le développement $\mathrm{du}$ sentiment national. Il mentionne l'idée de laïcité, supposément promue par le khédive Ismail (1863-1879), comme une notion-clé de la modernité. Masquée par les institutions islamiques orthodoxes mises en place par le khédive, la laïcité est, selon Warburg (ibid.: 7-8), étroitement liée aux ambitions modernisatrices d'Ismaïl. 
31 Une décennie plus tard, l'historien soudanais Hassan Ahmed Ibrahim (2001: 217-218) semble calquer sa lecture de la modernité au Soudan sur le modèle des théories de la modernisation. Il reprend le refrain désormais connu: l'histoire contemporaine soudanaise débute avec la conquête ottomano-égyptienne de 1820-1821 car la Turkiyya provoque l'unification politique du Soudan et entame un processus de modernisation, lequel se traduit avant tout par des innovations techniques et l'amélioration des opportunités commerciales à travers le Soudan. L'État est graduellement laïcisé sous l'impulsion de Mehmet Ali et de ses successeurs. Au processus de modernisation s'ajoute une tendance à l'occidentalisation, avec la présence d'un nombre croissant d'Européens au Soudan. Les fonctionnaires européens du gouvernement ottomano-égyptien ${ }^{17}$ engendrent des tensions au sein de la société soudanaise et des sentiments hostiles à l'égard des Européens et des étrangers en général (ibid. : 220). La modernité est ainsi associée à une époque durant laquelle une domination étrangère (ottomano-égyptienne) crée une entité politique unifiée (le Soudan moderne) et la transforme à l'aide de techniques et d'idées importées d'Europe.

Publié il y a une décennie, l'ouvrage de Heather Sharkey $(2003: 10,127)$ sur la formation d'une élite nationaliste nord-soudanaise à l'époque coloniale regorge de références à la modernité. L'historienne américaine emploie le terme de deux manières distinctes dans le contexte du Soudan anglo-égyptien (1899-1956). Il s'agit, d'une part, d'un ensemble de processus qui renvoient à l'histoire européenne. La modernité, c'est le progrès social fondé sur la science et la raison ; c'est le développement de l'éducation, de la santé et des technologies selon des modèles occidentaux. Vêtements européens, cinéma, plomberie, imprimerie, photographie: ces éléments disparates sont autant de mécanismes et de modalités typiques de la modernité auxquels les élèves nord-soudanais du Gordon College ont un accès privilégié (ibid.:65). L'enseignement institué par les États coloniaux à travers l'Afrique et l'Asie produit une élite "moderne ", dont la culture commune se manifeste par une bonne connaissance de l'anglais, le port d'habits européens, une expérience professionnelle au sein de l'administration coloniale et un engagement nationaliste (ibid.: 40). D'autre part, Sharkey utilise l'adjectif «moderne» au sens temporel de " contemporain », par exemple pour qualifier la littérature arabe, la nation, les frontières et la pensée soudanaise $\mathrm{du} \mathrm{xx}^{\mathrm{e}}$ siècle (ibid. : 51, 124, 125, 133). La modernité dans ces deux acceptions est employée en tant que catégorie d'analyse, c'est-à-dire comme un outil conceptuel. Toutefois, Sharkey se réfère également à la modernité en tant que catégorie mobilisée par les acteurs historiques eux-mêmes. Les Nord-Soudanais formés au Gordon College invoquent la modernité lorsqu'ils défendent ou revendiquent l'innovation, le port de vêtements occidentaux, un statut social élevé, un emploi de bureau, le progrès social et technique, la science occidentale et des attitudes «progressistes » (ibid. : 47, 50, 54, 132, 114, 129). Pour les administrateurs britanniques, l'éducation et les techniques européennes sont des signes incontournables de la « modernité » (ibid. : 53, 71, 93).

Bien qu'elle n'inscrive pas son propos dans un discours de la modernisation, sharkey concocte la modernité avec des ingrédients semblables à ceux utilisés par d'autres soudanistes avant elle. Cependant, elle tente de substituer à l'idée d'une modernisation exogène un processus d'indigénisation de la modernité (ibid.:11), tentative peu convaincante car insuffisamment étayée. Sharkey se distingue surtout de ses prédécesseurs par un usage double de la notion de modernité, en tant que catégorie analytique et en tant que catégorie normative. Cet usage est pourtant problématique car 
il demeure implicite ; la distinction entre ces deux types de catégories, essentielle dans toute démarche réflexive en sciences humaines et sociales, est souvent floue, non explicitée (ibid. : 65) ${ }^{18}$. En outre, l'absence de référence à des sources primaires pour démontrer l'usage du terme "modernité » par les acteurs historiques (comme une catégorie normative) affaiblit l'argumentaire général (ibid.: 50$)^{19}$. Sharkey évoque bel et bien l'existence d'un débat historiographique récent sur la modernité (ibid. : 97-98), mais sans véritablement prendre position. Cette posture peu engagée ne permet pas de faire de la modernité un concept réellement opératoire pour l'analyse des sociétés humaines.

Certains historiens soudanais ont pris le contrepied des travaux qui épousent plus ou moins fidèlement, plus ou moins consciemment, les théories de la modernisation. Les interprétations de Muhammad Sa î̂d al-Gaddâl et d"Abd al-Azîz Husayn al-Sâwî s'appuient sur les théories de la dépendance.

\section{L'irruption de la modernité ou la périphérisation dans le système mondial}

Les théories de la dépendance ont été élaborées dans les années 1960 et 1970 par des économistes et sociologues argentin (Raúl Prebisch), allemand (Andre Gunder Frank) et américain (Immanuel Wallerstein) en réaction aux théories de la modernisation, jugées excessivement européocentriques et inadéquates pour décrire le monde contemporain. À l'instar de celles-ci, les théories de la dépendance sont fondées sur une vision dichotomique de l'ordre mondial, divisé entre pays riches/industrialisés/dominants et pays pauvres/sous-développés/dépendants. Les pays du premier groupe sont membres de l'Organisation de coopération et de développement économiques (OCDE) et majoritairement occidentaux; ceux du second groupe sont répartis entre l'Amérique latine, l'Afrique et l'Asie.

Bien que plurielles, les théories de la dépendance reposent sur certaines prémisses communes :

- les pays pauvres fournissent des ressources naturelles et une main-d'œuvre bon marché aux pays riches, qui leur transmettent en retour des technologies obsolètes et leur vendent des produits manufacturés. Les sociétés des pays industrialisés accèdent ainsi à un niveau de vie très élevé ;

- les pays riches perpétuent activement - consciemment ou non - l'état de dépendance des pays pauvres. Cette dépendance est économique, financière, politique et médiatique ;

- les tentatives des pays pauvres de lutter contre cette dépendance se soldent souvent par des sanctions économiques ou des invasions militaires de la part des pays riches (Ferraro 2008). Historiquement, selon ces théories, la domination des pays occidentaux sur le reste du monde s'est construite au $\mathrm{XIX}^{\mathrm{e}}$ siècle à travers la Révolution industrielle, la formation d'immenses empires coloniaux européens et l'expansion du capitalisme ${ }^{20}$.

Dans une courte étude consacrée à l'évolution de l'islam soudanais au XIX ${ }^{e}$ siècle, alGaddâl (1985 : 49) envisage la modernité dans une perspective qui rappelle les théories de la dépendance. Le xIX ${ }^{e}$ siècle représente une période "remarquable " pour le Soudan, car sa structure socio-économique a subi de profonds changements et le pays est devenu une proie du colonialisme. La terminologie employée par al-Gaddâl ne fait aucun doute quant à l'orientation marxiste de son approche historique: «la montée d'une classe moyenne marchande ", "l'intégration dans un marché capitaliste mondial en pleine expansion ", «les intérêts des masses rurales opprimées ", «l'intensification de la lutte des classes 
dans la société musulmane ", "la défaite de la classe marchande » (ibid.: 49-55) ${ }^{21}$. La modernité est donc le processus par lequel le Soudan est intégré au système capitaliste mondial et envahi par des impérialistes étrangers (ottomano-égyptiens et britanniques). La révolution mahdiste, en tant que réaction à la domination ottomano-égyptienne, est considérée comme un «mouvement de libération nationale incapable de s'exprimer de façon laïque » (ibid. : 54).

Dans un ouvrage intitulé Ta'rîkh al-Sûdân al-Hadîth: 1820-1955 (L'histoire du Soudan contemporain : 1820-1955), al-Gaddâl souligne que :

«La lutte contre la domination étrangère et les tentatives du peuple soudanais de se libérer de son emprise constituent l'élément qui a façonné le cours de l'histoire du Soudan contemporain. "(al-Gaddâl $1993: 12$ )

Ce n'est pas parce qu'il met en évidence le couple impérialisme-nationalisme comme composante significative de la modernité qu'al-Gaddâl renonce à son analyse marxiste. L'interaction de facteurs exogènes et endogènes engendre des processus qui transforment violemment le Soudan à l'époque contemporaine. D'une part, l'invasion étrangère provoque l'unification politique du Soudan et son intégration croissante dans le marché capitaliste mondial. D'autre part, des changements profonds dans la structure socioéconomique du pays, de nature endogène au départ, sont "détournés " par l'agent extérieur (l'impérialisme ottomano-égyptien) pour mettre le Soudan au service du marché capitaliste (ibid. : 17).

Ainsi, bien qu'al-Gaddâl date les débuts de la modernité aux premières phases du règne ottomano-égyptien, à l'instar des historiens évoqués précédemment ${ }^{22}$, le sens de cette modernité n'est pas celui d'une modernisation vers un certain progrès. Il s'agit plutôt de la transformation du Soudan, par le biais de l'impérialisme étranger, en une nouvelle périphérie du système capitaliste mondial.

Al-Sâwî (1994: 19) reconnaît au gouvernement ottomano-égyptien sa contribution à l'unification territoriale du Soudan, l'établissement d'un système politique approximant un État moderne, le développement de moyens de communication et de techniques agricoles «avancés » et la diversification de l'activité économique. Il s'agit cependant d'un système étranger essentiellement oppressif et exploiteur. C'est l'État mahdiste qui, en revitalisant et en activant la "cohésion nationale ", a joué un rôle décisif dans la formation du Soudan moderne (ibid. : 22). Hélas, selon l'historien et journaliste soudanais, la dernière décennie du xix ${ }^{e}$ siècle est le moment du partage colonial de l'Afrique, produit direct de la "maturation du phénomène capitaliste »: la défaite du mahdisme est inévitable face au modèle occidental industriel (ibid. : 24-25). À partir de là, l'histoire du Soudan est celle d'une lutte contre le «visage ravageur du capitalisme occidental». Le système colonial ne secrète qu'une modernisation « marginale », qui force le Tiers monde à emprunter un chemin long et tortueux pour obtenir son indépendance (ibid. : 25).

\section{Le Soudan et la modernité : cas particuliers}

42 À côté d'études qui approchent globalement le problème de la modernité au Soudan, des analyses traitent de la question à travers des cas ou sujets très particuliers. De par leur objet d'étude mieux circonscrit, ces études permettent des conceptualisations plus fines ou plus complexes de la modernité. Malgré une grande variété de thèmes et d'approches épistémologiques, on peut y distinguer deux catégories principales : les travaux qui font 
apparaitre la modernité sous la forme d'agents individuels et ceux qui l'exposent sous la forme de systèmes. Au-delà des différentes facettes de la modernité, nous verrons comment le vêtement que les spécialistes en sciences sociales font porter à la modernité (individuel ou systémique) se répercute sur le sens même de la catégorie modernité.

\section{Agents individuels de modernité}

La modernité se présente sous la forme d'agents individuels dans des études portant sur le développement de la médecine tropicale et l'activité de missionnaires chrétiens au Soudan. J'évoquerai deux exemples de chaque catégorie afin de montrer que des études portant sur le même sujet peuvent conceptualiser la modernité selon des modalités très diverses.

Le médecin et enseignant Alexander Cruikshank (1985: 85-100) expose comment un système de santé moderne fut établi au Soudan entre la fin du XIX ${ }^{e}$ siècle et la première moitié du xxe siècle. Il survole le développement d'infrastructures médicales telles que les hôpitaux de Khartoum, d'Atbara et de Port Sudan, l'école pour sages-femmes d'Omdurman et l'école de médecine Kitchener. Il décrit également la lutte contre la lèpre et les épidémies qui sévissent dans de nombreuses régions du Soudan à l'époque (méningite cérébrospinale, fièvre jaune, variole, maladie du sommeil). Ces processus sont induits par l'action d'individus, dont la contribution à l'établissement de services médicaux au Soudan est largement félicitée par l'auteur. Ainsi, Mabel Wolff, une sagefemme ayant exercé en Égypte, se vit confier la difficile mission de diriger l'école pour sages-femmes fondée en 1922 à Omdurman. Selon Cruikshank (ibid. : 89), c'est grâce à sa bonne connaissance de la langue arabe et à son sens de l'humour qu'elle réussit à développer et à dynamiser l'école. Quant à Oliver Atkey, directeur du Sudan Medical Service (SMS) dès 1922, il fut responsable de la gestion de l'école de médecine Kitchener, fondée en 1924 à Khartoum. Atkey envoya Cruikshank au pays des Azande (province d'Equatoria) afin d'y créer un centre de soins pour les lépreux. Ces exemples suffisent à illustrer l'importance, dans la vision de Cruikshank, de l'action individuelle, capable de répandre des éléments de modernité - avant tout des techniques médicales - là où ils n'existaient pas auparavant.

Outre l'importance des agents individuels en tant que catalyseurs de la modernisation, l'étude de Cruikshank (ibid. : 100) défend une interprétation proche des théories de la modernisation discutées plus haut. Refusant de définir la modernité au sens strict de « la poudre à canon, l'imprimerie et la religion protestante $\aleph^{23}$, Cruikshank voit la modernisation comme :

«La capacité d'un pays ou d'une nation à maintenir toutes les caractéristiques importantes et pertinentes du moment actuel [...] ceci implique un niveau éducatif et culturel ainsi que des compétences physiques et mentales permettant de comprendre, d'apprécier et de contribuer aux caractéristiques valables de l'homme moderne. "(ibid.)

Malgré la relative flexibilité de cette conception, Cruikshank semble tout de même associer la modernisation à une occidentalisation (surtout dans les domaines technique et scientifique), qui permettrait au Soudan d'avoir sa place dans le monde moderne. Cette « entrée » dans le monde moderne représente selon lui (ibid.) une avance, un progrès.

Relevant également du domaine médical, l'étude de Heather Bell (1998 : 293-312) porte sur la formation des sages-femmes et la pratique de l'excision dans le Soudan anglo- 
égyptien de l'Entre-deux-guerres. Loin d'être présentée comme un simple processus de modernisation, l'introduction de la médecine occidentale au Soudan paraît constituer l'une des facettes d'un phénomène bien plus complexe: l'interaction entre différents types de médecine (occidentale et traditionnelle) et entre des sociétés (britannique et soudanaise) fondées sur des systèmes de valeurs divergents. Le rôle des sœurs Mabel et Gertrude Wolff (qui dirigèrent l'école pour sages-femmes d'Omdurman durant près de vingt ans) ne se réduit pas à semer les graines de la modernité sur une terre «traditionnelle» :

« Dans la construction de la respectabilité de leurs élèves potentielles, leur choix de la langue d'enseignement, leurs stratégies d'assermentation de sages-femmes traditionnelles et formées ainsi que dans leur approche de l'excision, les femmes britanniques qui dirigeaient l'école pour sages-femmes [Midwifery Training School, MTS] [...] étaient constamment en train de négocier avec la culture soudanaise et de se heurter aux limites du pouvoir (médical) colonial britannique. Alors que la formation et la pratique de sages-femmes incorporaient les sœurs Wolff et des sages-femmes soudanaises dans le champ d'action de l'État colonial, elles demeuraient marginalisées au sein de cet État, dépourvues d'autorité, de statut et de rémunération à cause de leur sexe, de leur classe et de leur profession. »(ibid. : 312)

L'article de Bell, en combinant une étude historique précise avec une approche anthropologique axée sur le genre, permet de concevoir une image plus complexe, nuancée et critique de la modernité au Soudan. Plutôt que le réceptacle passif de techniques et de notions importées d'Europe, le Soudan contemporain est un lieu de rencontre entre différentes cultures, traditions et visions du monde, qui négocient entre elles de façon permanente. Bell (ibid. : 293) contribue également à tordre le coup à une idée reçue encore largement répandue, celle qui postule qu'au temps des empires coloniaux, les praticiens de la médecine occidentale en Afrique subsaharienne étaient des Européens pour la plupart. Dans le Soudan anglo-égyptien, la majorité des médecins étaient syriens, égyptiens ou soudanais, tandis que leurs assistants, les infirmières et les sages-femmes étaient soudanais.

Les études sur les activités des missionnaires chrétiens au Soudan durant le Condominium anglo-égyptien conçoivent la modernité autrement. Ainsi, chez l'anthropologue John Burton (1985), la modernité apparaît au Sud-Soudan comme un conflit entre une conversion religieuse (l'action des missionnaires) et ce qu'il nomme une " conversion laïque " (la politique du régime colonial britannique). Insistant sur le fait que les différentes missions chrétiennes ${ }^{24}$ étaient totalement subordonnées au gouvernement colonial, Burton (ibid. : 361) met en évidence la façon dont celui-ci utilisa les missionnaires pour mettre en œuvre sa politique de conversion séculière. Cette politique consistait à créer une élite indigène formée à un régime laïc, autoritaire et représentatif. Que les membres de cette élite aient suivi une formation initiale religieuse chez les missionnaires, pouvait contribuer à ériger « un rideau idéologique entre le Sud et le Nord-Soudan musulman » (ibid.). Avec la proclamation de la Southern Policy en $1930^{25}$, le régime colonial britannique modifia son attitude à l'égard des peuples indigènes qui devaient désormais être traités comme

«une série d'unités raciales ou tribales délimitées, dont la structure et l'organisation sont fondées, dans la mesure où les besoins d'équité et de bonne gouvernance le permettent, sur les coutumes indigènes, les pratiques et les croyances traditionnelles. »(ibid.: 362) 
50 jeunes indigènes à l'étude de matières relatives au droit et à l'administration. Les missionnaires furent ouvertement critiqués par des fonctionnaires du régime colonial qui les accusaient de détruire les "coutumes tribales » qu'eux-mêmes avaient contribué à créer. Burton (ibid. : 363) voit ainsi la mission civilisatrice et modernisatrice de la GrandeBretagne au Sud-Soudan comme un paradoxe: d'un côté, les missionnaires étaient employés pour instruire les populations locales d'une façon qui convenait aux colonisateurs mais, de l'autre côté, ils étaient blâmés lorsqu'ils avaient accompli cette tâche.

51 Dans un article consacré aux activités missionnaires dans les monts Nouba au cours des années 1930 et 1940, Justin Willis (2003 : 32) développe également une réflexion sur les interactions entre missionnaires et agents du gouvernement colonial. Il constate l'incapacité de la Church Missionary Society à fonder une communauté chrétienne locale et explique cet échec par l'attitude ambivalente des missionnaires à l'égard du couple tradition-modernité. En effet, les missionnaires avaient une vision très romantique des Noubas, considérés comme un peuple

«intouché par le fléau de la modernité, prêt à passer directement du paradis à l'église chrétienne [...] l'ennemi [pour les missionnaires] n'était pas l'ignorance et l'état arriéré, mais "l'impact soudain de la vie et de la civilisation occidentales", qui avaient un dangereux effet de "désintégration". »(Cash 1933 : 16, 18)

D'une part, les missionnaires tentèrent de créer une école privilégiant ce qu'ils considéraient comme les éléments-clés de la «culture Nouba » : les filles, par exemple, n'étaient pas autorisées à porter des vêtements et elles devaient s'adonner à la cuisine et à l'agriculture (Willis 2003: 50) ${ }^{26}$. D'autre part, les missionnaires donnèrent accès à la modernité sous la forme de services médicaux. Ils ne parvenaient pas à réconcilier leur conception essentialiste des Noubas avec la spiritualité éclectique et diversifiée de cette population. Les chefs locaux ne partageaient pas la croyance des missionnaires selon laquelle la «tradition » constituait le seul fondement possible de l'autorité. Pour eux, les représentants de la mission chrétienne et leurs pratiques représentaient également des sources potentielles de richesse et de santé, parmi d'autres sources (ibid. : 47). Ainsi, Willis (ibid. : 33) évalue l'épisode de la Church Missionary Society dans les monts Nouba comme

« un combat incertain entre des missionnaires enfermés dans une vision simpliste et dichotomique de la tradition et du changement, un État colonial tiraillé entre les exigences contradictoires de l'idéologie et de la pratique administrative, et une population africaine constamment à la recherche de nouvelles sources de richesse matérielle et de pouvoir spirituel. »

Oscillant entre une perspective "traditionaliste» voulue anti-modernisatrice et une approche modernisatrice prônant une certaine occidentalisation, les missionnaires des monts Nouba s'empêtrèrent dans leurs propres contradictions. Ils échouèrent à créer une communauté de chrétiens africains qui préserverait la «tradition» nouba tout en épousant les croyances et rites anglicans.

Ces quelques études suggèrent différentes conceptions de la modernité à travers les actions d'agents individuels. Ceux-ci sont parfois décrits comme le moteur principal d'une modernisation lisse qui, sur le terrain soudanais, aurait rencontré peu d'obstacles d'ordre culturel (analyse de Cruikshank). Dans d'autres cas, des individus enjambent les fossés qui séparent les conceptions et techniques européennes des pratiques soudanaises locales afin de négocier des usages nouveaux et hybrides (comme chez Bell, par exemple).

Cahiers d'études africaines, 208 | 2012 
Enfin, la modernité est aussi l'hésitation et l'inconsistance mêmes qui caractérisent la politique coloniale britannique et l'activité des missionnaires au Soudan (études de Burton et Willis). Une attitude ambivalente à l'égard des populations dominées, imprégnée de romantisme et façonnée par les besoins administratifs du moment, paraît en effet constituer l'un des signes distinctifs de la modernité dont les colonisateurs et les missionnaires se réclamaient.

D'autres études spécifiques évoquent la modernité en tant que système. Plutôt que de mettre en scène des individus associés à la modernité, elles dépeignent le mouvement de forces - parfois très abstraites -, de processus ou de systèmes qui se succèdent les uns aux autres ou qui s'entrechoquent brutalement.

\section{Systèmes de modernité}

Trois études retenues habillent la modernité d'un vêtement "systémique ». Très distinctes dans leurs approches - historique marxiste, anthropologique ou juridique -, elles traitent de sujets aussi différents que le rôle social des femmes dans l'Ouest du Soudan, le mouvement mahdiste et la question de la liberté religieuse dans le Soudan de la fin du $\mathrm{Xx}^{\mathrm{e}}$ siècle.

Lidwien Kapteijns (1985 : 57-72) analyse l'évolution du rôle social des femmes dans la région des sultanats du Ouaddaï (1675-1909) et du Darfour (1650-1874, 1898-1916) dans une perspective de longue durée. Une distinction claire est instaurée entre la période " précoloniale » (qui dure jusqu'à la conquête ottomano-égyptienne du Soudan en 1820) et les périodes "coloniales et postcoloniales" (règne ottomano-égyptien, Mahdiyya, Condominium anglo-égyptien et Soudan indépendant). Adoptant une approche de genre teintée de marxisme, Kapteijns associe la modernité à de profondes transformations socio-économiques. Selon elle, «le capitalisme commercial commença à remplacer le féodalisme dans la vallée du Nil en 1800 environ et plus rapidement après la conquête turco-égyptienne de $1821 »$ (ibid. : 66). La modernité pénètre dans la région du Ouaddaï/ Darfour sous une forme intrusive, celle d'un nouveau système économique importé de la vallée du Nil et porteur d'une certaine idéologie islamique. Sous la plume de Kapteijns ( ibid. : 66-67), ces changements économiques et idéologiques constituent un processus qui transcende les périodes coloniales et postcoloniales. Ottomano-Égyptiens, mahdistes et Britanniques représentent des étrangers qui étendent l'influence politique, économique et idéologique de la vallée du Nil dans l'Ouest du Soudan ${ }^{27}$.

La conséquence de cette intrusion fut, selon Kapteijns, la désintégration de l'organisation politique et économique traditionnelle de la région. Les femmes du Ouaddaï/Darfour perdirent une part importante de l'indépendance économique, de la responsabilité légale et de la liberté sociale dont elles jouissaient durant la période précoloniale. Elles se virent contraintes à adopter les codes sociaux et religieux de l'islam tel qu'il était pratiqué dans la vallée du Nil. Les migrations de travail en direction des villes provoquèrent l'éclatement de la cellule familiale et les mères se virent obligées de pourvoir seules aux besoins vitaux de la famille. Dans les zones rurales, les femmes tentèrent d'imiter le mode de vie des nouvelles classes moyennes, considéré plus islamique que le mode de vie traditionnel. Elles se mirent à exciser leurs filles et à les confiner à l'intérieur de la maison (ibid. : 67-68).

L'analyse de Kapteijns (ibid. : 57) se conforme au modèle des théories de la dépendance en y ajoutant toutefois un soupçon de féminisme. La modernité vécue par la région du 
Ouaddaï/Darfour apparait comme une transformation brutale et profonde des structures socio-économiques locales. Ce changement a pour moteur le capitalisme industriel, répandu au moyen d'agents "extérieurs » qui, dans le cas examiné, exploitent les inégalités économiques institutionnalisées de l'islam au profit d'une nouvelle classe moyenne d'hommes. Transformé, le Soudan occidental devient «une périphérie économique, culturelle et politique de l'État colonial et postcolonial».

Dans une approche anthropologique du mouvement mahdiste soudanais, Kazuo Ohtsuka (1997 : 30) s'attache à réfuter l'idée selon laquelle ce mouvement, parce que religieux et salafiste, est nécessairement traditionaliste et anti-moderniste. Les arguments qu'il emploie pour avancer que le mahdisme soudanais constitue une forme modernisée d'islam paraissent cependant contradictoires. Premièrement, il montre à travers une analogie avec le christianisme que l'aspect salafiste du mouvement correspond à des caractéristiques typiques du protestantisme: monothéisme strict, puritanisme, importance de la révélation scripturaire, égalitarisme entre croyants, absence d'une médiation particulière ou d'une hiérarchie, minimisation de l'extravagance rituelle ou mystique, observance de règles (ibid. : 26-27) ${ }^{28}$. S'inspirant de la théorie de Max Weber qui lie étroitement l'éthique protestante à l'esprit du capitalisme, facteur de la modernité, Ohtsuka conclut que le mahdisme peut être considéré comme un type d'islam modernisé.

Deuxièmement, il affirme qu'un grand nombre d'islamistes, tel que le pionnier du mouvement salafiste Muhammad 'Abduh, bénéficièrent d'une instruction "moderne " (de type occidental) plutôt que musulmane «traditionnelle» (ibid.: 30). Le chef du mouvement mahdiste Muhammad Ahmad fut une exception à ce titre. Selon Ohtsuka, sa formation «traditionnelle " (théologie musulmane et soufisme) reflète le fait que le Soudan, contrairement à l'Égypte, n'avait pas encore été incorporé dans le «système mondial moderne " au milieu du xIX siècle. Ces conditions historiques contraignirent le mahdî à articuler son idéologie dans le langage de l'islam, bien qu'il ait partagé avec d'autres islamistes une vision critique de l'islam tel qu'il était pratiqué dans la société. Ainsi, il rejetait l'innovation (bid'a) et cherchait à exclure de la foi les éléments perçus comme "non islamiques» (ibid.: 31-32). Pour le chercheur japonais, le caractère révolutionnaire du mouvement mahdiste en fait un mouvement « moderne ».

Finalement, Ohtsuka (ibid.: 32) interprète l'apparent traditionalisme du mahdisme comme un traditionalisme " nouvellement inventé » (au sens où l'entend Eric Hobsbawm [1983]) et la société idéale envisagée par le mouvement comme une "communauté imaginée » (selon la terminologie de Benedict Anderson). Le fait que les mahdistes se soient opposés à certaines traditions propagées par les 'ulamâ' conservateurs est un signe de leur modernité.

63 La faiblesse suivante est décelable dans l'argumentaire d'Ohtsuka: il semble voir dans chaque aspect du mahdisme une manifestation de la modernité du mouvement, jusqu'à insinuer que tout et son contraire peuvent être considérés comme modernes. Par exemple, la ressemblance avec le protestantisme fait du mahdisme un courant moderne, mais l'éducation musulmane "traditionnelle » du mahdî ne réduit pas la dimension moderne du mouvement. Ohtsuka a toutefois le mérite de repenser la dichotomie conventionnelle entre moderne et traditionnel. Il nous invite à remettre en question «la terminologie habituelle qui identifie la "modernisation" à l'occidentalisation" ou à la "sécularisation" " (Ohtsuka 1997: 32). Les islamistes ont ainsi leur manière propre de «moderniser» la société, dans la mesure où ils la préparent à faire face à des pays étrangers, non musulmans. 

de la liberté religieuse dans le Soudan contemporain. Son auteur, Hervé Bleuchot (1995: 143), affirme qu'eut égard à la liberté religieuse deux systèmes juridiques sont possibles, le système antique et le système moderne. Le premier se fonde sur les principes suivants : l'État dirige les consciences individuelles selon les normes de la religion dominante ; l'État contrôle la religion dominante et les religions minoritaires; la religion dominante contrôle l'État (ils se contrôlent donc réciproquement); l'État et la religion dominante contrôlent l'ensemble de la vie sociale (confessionnalisation de la société). Le système moderne s'appuie sur des principes inverses : liberté de conscience sur le plan individuel, liberté des cultes au niveau collectif, sécularisation de l'État et sécularisation de la vie sociale (ibid.).

L'étude de Bleuchot vise à montrer qu'avec l'islamisation du droit en $1983^{29}$, le Soudan passa du système moderne au système antique. S'appuyant sur des textes juridiques tels que le Self-Government Statute (1953), la Constitution de 1974 et le code pénal (introduit en 1899 puis amendé en 1925, 1955 et 1974), le chercheur montre que jusqu'en 1983, le système soudanais était «moderne » en ce qu'il respectait la liberté religieuse. Comment put-il brutalement céder la place à un système « antique » en 1983 ? Parce que le système « moderne » avait été

«établi dans une société qui n'en avait pas compris la valeur (à l'exception d'un petit nombre) et ne fit rien pour le défendre [...]. Les partisans de sa chute n'eurent donc aucune peine à le faire disparaitre. »(ibid. : 157)

La modernité épouse ici la forme d'un système juridique destiné à préserver certains principes qui régulent la vie en société. Chez Bleuchot, le principe de la liberté religieuse est central. Il est directement hérité de la philosophie des Lumières ainsi que de ses corollaires, la laïcisation de l'État et la sécularisation de la société. étudiés (politique, économie, techniques, culture, religion, genre, colonialisme, médecine, droit), la grande majorité des soudanistes, dans leur évocation de la modernité, font écho aux conceptions européennes décrites plus haut. Ainsi Hill, Holt, Daly, Ibrahim et Sharkey soulignent les progrès techniques et l'impact culturel de la présence européenne au Soudan, alors que Warburg, al-Gaddâl et Kapteijns mettent en relief les transformations socio-économiques de la région - en particulier l'expansion du capitalisme. La plupart mentionnent la création d'une entité politiquement unifiée comme l'un des éléments-clés de la modernité. Cruikshank évoque celle-ci à travers le prisme du progrès médical, tandis que Bleuchot l'associe aux processus de laïcisation étatique et de sécularisation sociétale. Bell, Burton, Willis et Ohtsuka ébauchent une image de la modernité plus floue mais plus nuancée.

70 Les études de Bell, de Burton et de Willis font apparaître la modernité sous la forme d'attitudes coloniales inconsistantes et ambivalentes à l'égard des colonisés. Elles montrent que les acteurs identifiés à la puissance coloniale britannique (administrateurs, directrices de l'école pour sages-femmes) ou à des organisations occidentales (missionnaires) n'épousaient pas automatiquement la perspective «classique» d'une 
modernisation bienveillante, synonyme d'importation des techniques et normes européennes en territoire colonisé. Confrontés à des problèmes moraux, pratiques et administratifs, ces acteurs devaient constamment redéfinir leur mission, leurs objectifs et leur attitude à l'égard de la modernité occidentale.

Ohtsuka remet franchement en cause le concept de modernisation employé au sens d'occidentalisation. Il doute de la pertinence du concept utilisé de cette manière, critiquant son inadéquation avec la réalité. En effet selon lui, la modernisation (occidentalisation) laisse de côté des phénomènes de modernisation qui n'impliquent pas d'occidentalisation, ou seulement partiellement. Malheureusement, Ohtsuka (1997 : 33) ne propose aucune définition alternative du concept de modernisation. Il se contente de déclarer qu'il n'est le synonyme exact ni de l'occidentalisation ni de la sécularisation telles qu'elles sont conçues en Occident.

Seule l'étude de Sharkey éclaire la manière dont la modernité a pu fonctionner en tant qu'outil de revendication dans le Soudan colonial, ouvrant timidement la voie à une conceptualisation de la modernité en tant que catégorie « indigène $»^{30}$, mobilisée par des acteurs sociaux dans des circonstances historiques particulières. Le problème de la nécessaire distinction entre catégories normatives et analytiques demeure cependant irrésolu, n'ayant jusqu'à présent pas reçu l'attention qu'il mérite dans le champ des études soudanaises.

Conçue comme un processus d'européanisation, la modernisation conserve sans doute une valeur heuristique, mais ses trop nombreuses dérives - dénoncées à juste titre vers des interprétations déterministes teintées d'évolutionnisme social l'ont condamnée en tant que concept académiquement valide. Que se passe-t-il si, à l'instar d'Ohtsuka, de certains sociologues et des subalternistes, l'on adopte un argumentaire postcolonialement correct en postulant l'existence de modernités "multiples", « alternatives », " polycentriques » ou « hybrides » $?^{31}$ Le brouillard conceptuel s'épaissit au lieu de se dissiper. Ces modernités en viennent à désigner tout événement ou processus localisé dans le flux des cinq cents dernières années. Comment sortir de ce qui paraît être une impasse épistémologique?

74 La démarche de Cooper $(2005: 133,146)$ suggère une piste possible, celle qui consiste à abandonner la modernité comme pseudo catégorie analytique pour ne plus la considérer que comme une catégorie normative, de pratique. Les discours de la modernité peuvent être utilement examinés non seulement en tant qu'instrument de revendication, comme le propose le chercheur américain, mais également en tant que mécanisme de légitimation d'idées, d'attitudes et de pratiques spécifiques ${ }^{32}$.

Une autre option, moins radicale mais peut-être pas moins féconde, est de continuer à utiliser le concept de modernité comme catégorie analytique, mais en prenant soin de clarifier son sens au préalable. Un choix s'impose alors: emploie-t-on le terme comme une simple référence au présent, à une période actuelle qui diffère d'époques antérieures en fonction de critères que l'on précisera? Désigne-t-on par là une portion spécifique du passé historique européen? Ou encore, la modernité renvoie-t-elle à des processus politiques, économiques, sociaux, culturels ou intellectuels déterminés? Ces différents usages semblent légitimes à partir du moment où ils résultent d'un choix conscient, explicité et justifié par rapport à l'objet d'étude. En tant qu'êtres pensants par et grâce au langage, nous construisons inévitablement des catégories pour méditer sur le monde présent et passé. Heuristiquement parlant, il est certainement plus utile d'affiner leurs significations que de les rendre sans cesse plus fluides et englobantes, procédé en vogue 
dans un univers académique qui peine à résoudre l'un des paradoxes du postmodernisme: bien que dépassée, voire récusée, la «modernité» demeure une ressource discursive centrale pour des hommes et des femmes qui ne parviennent pas à définir leur propre époque sans s'y référer.

\section{BIBLIOGRAPHIE}

ABU-LUGHOD, L. (ED.)

1998 - Remaking Women : Feminism and Modernity in the Middle East, Princeton, Princeton University Press.

ALIDOU, $\mathrm{O}$.

2005 - Engaging Modernity : Muslim Women and the Politics of Agency in Postcolonial Niger, Madison, University of Wisconsin Press.

ANDERSON, B.

2002 [1983] - L'imaginaire national. Réflexions sur l'origine et l'essor du nationalisme, Paris, La

Découverte.

APPADURAI, A.

1996 - Modernity at Large : Cultural Dimensions of Globalization, Minneapolis, University of Minnesota Press.

ARON, R.

1969 - Les désillusions du progrès : essai sur la dialectique de la modernité, Paris, Calmann-Lévy.

AWAH, P. K. \& PHILLIMORE, P.

2008 - « Diabetes, Medicine and Modernity in Cameroon », Africa, 78 (4) : 475-495.

BAKHÎT, J. M. A.

1987 - Al-Idâra al-Barîtâniyya wa-l-Haraka al-Wataniyya fî al-Sûdân, 1919-1939, Khartoum, Al-Matba‘a al-‘Arabiyya li-l-Ta'lîf wa-l-Tarjama.

BARTOlOVich, C. \& lazARUS, N. (EDS.)

2002 - Marxism, Modernity, and Postcolonial Studies, Cambridge, Cambridge University Press.

BELL, H.

1998 - « Midwifery Training and Female Circumcision in the Inter-War Anglo-Egyptian Sudan », Journal of African History, 39 (2) : 293-312.

BLEUCHOT, $\mathrm{H}$.

1995 - « La liberté religieuse au Soudan : du système moderne au système antique », Annuaire de

l'Afrique du Nord, 34 : 143-173.

BOURETZ, P.

1987 - «"Progrès" ", in P. ORY (dir.), Nouvelle histoire des idées politiques, Paris, Hachette : 369-378.

BURTON, J. W.

1985 - «Christians, Colonists and Conversion : A View from the Nilotic Sudan », Journal of Modern African Studies, 23 (2) : 349-369. 
CĂLINESCU, M.

1987 - Five Faces of Modernity : Modernism, Avant-Garde, Decadence, Kitsch, Postmodernism, Durham, Duke University Press.

CARLYLE, T.

2010 - Critical and Miscellaneous Essays, in H. D. TRAILl (ed.), The Works of Thomas Carlyle, vol. 26, Cambridge, Cambridge University Press. Fac-similé de l'édition de 1899 édité par Chapman and Hall, London.

CASH, W. W.

1933 - The Nubas Calling: A Challenge to Pioneer Missionary Adventure among Sudan Hill Tribes, London, Church Missionary Society.

CHAKRABARTY, D.

2000 - Provincializing Europe : Postcolonial Thought and Historical Difference, Princeton, Princeton University Press.

2002 - Habitations of Modernity : Essays in the Wake of Subaltern Studies, Chicago, University of Chicago Press.

CHAMBERS, I.

2008 - Mediterranean Crossings : The Politics of an Interrupted Modernity, Durham, Duke University Press.

CHAUDHURI, A.

2008 - Clearing a Space : Essays on Literature, India, and Modernity, Oxford, Peter Lang.

CIAFFA, J. A.

2008 - « Tradition and Modernity in Postcolonial African Philosophy », Humanitas, 21 (1/2) :

121-145.

COLLINS, R. O.

1967 - Egypt and the Sudan, Englewood Cliffs (N. J.), Prentice-Hall.

2008 - A History of Modern Sudan, Cambridge, Cambridge University Press.

COOPER, F.

2005 - Colonialism in Question : Theory, Knowledge, History, Berkeley, University of California Press. CRUIKSHANK, A.

1985 - « The Golden Age of Tropical Medicine and its Impact on the Modernization of the Sudan ", in M. W. DALY (ed.), Modernization in the Sudan, Essays in Honor of Richard Hill, New York, Lilian Barber Press : 85-100.

DALY, M. W.

1985 - «Introduction », in M. W. DALY (ed.), Modernization in the Sudan, Essays in Honor of Richard Hill, New York, Lilian Barber Press : 7-14.

DE JONG, F.

2005 - Masquerades of Modernity : Power and Secrecy in Casamance, Senegal, Bloomington, University of Indiana Press.

EISENSTADT, S. N.

2000 - « Multiple Modernities », Daedalus, 129 (1) : 1-29.

ELIAS, $\mathrm{N}$.

1996 [1984] - Du temps, Paris, Fayard. 
FERRARO, V.

2008 - « Dependency Theory : An Introduction », in G. SECONDI (ed.), The Development Economics Reader, London, Routledge : 58-64, <http://www.mtholyoke.edu/acad/intrel/depend.htm>.

FRANK, A. G.

1974 - « Dependence is Dead, Long Live Dependence and the Class Struggle », Latin American Perspectives, 1 (1) : 87-106.

FRIEDMAN, S. S.

2006 - « Periodizing Modernism : Postcolonial Modernities and the Space/Time Borders of Modernist Studies », Modernism/Modernity, 13 (3) : 425-443.

AL-GADDÂL, M. S.

1985 - « Religion in a Changing Socio-Political Structure : A Case Study of Islam in NineteenthCentury Sudan », in M. W. DALY (ed.), op. cit. : 49-56.

1993 - Ta'rîkh al-Sûdân al-Hadîth : 1820-1955, Khartoum, Sharikat al-'Amal li-l-Tibâ'a wa-l-Nashr.

GAONKAR, D. P. (ED.)

2001 - Alternative Modernities, Durham, Duke University Press.

GELLNER, E.

1969 - « The Pendulum Swing Theory of Islam », in R. ROBERTSON (ed.), Sociology of Religion, Harmondsworth, Penguin Books : 127-138.

1999 [1983] - Nations et nationalisme, Paris, Payot \& Rivages.

GOODY, J.

2004 - Capitalism and Modernity: The Great Debate, Cambridge, Polity Press.

HABERMAS, J.

1988 - Le discours philosophique de la modernité. Douze conférences, Paris, Gallimard.

HAROOTUNIAN, $\mathrm{H}$.

2001 - Overcome by Modernity: History, Culture, and Community in Interwar Japan, Princeton, Princeton University Press.

HENDRICKSON, H. (ED.)

1996 - Clothing and Difference : Embodied Identities in Colonial and Post-Colonial Africa, Durham, Duke University Press.

HERSCH, J.

1993 - L'étonnement philosophique : une histoire de la philosophie, Paris, Gallimard.

HILL, R. L.

1959 - Egypt in the Sudan, 1820-1881, London, Oxford University Press.

HOBSBAWM, E. J.

1990 - Nations and Nationalism since 1780 : Programme, Myth, Reality, Cambridge, Cambridge

University Press.

HOBSBAWM, E. J. \& RANGER, T. O. (EDS.)

1983 - The Invention of Tradition, Cambridge, Cambridge University Press.

HOLDEN, P.

2008 - Autobiography and Decolonization: Modernity, Masculinity, and the Nation-State, Madison, University of Wisconsin Press. 
HOLT, P. M.

1973 - « Modernization and Reaction in the Nineteenth-Century Sudan », in P. M. HOLT (ed.), Studies in the History of the Near East, London, F. Cass : 135-148.

HOLT, P. M. \& DALY, M. W.

2000 [1961] - A History of the Sudan from the Coming of Islam to the Present Day, London, Longman.

IBRAHIM, H. A.

2001 - « The Strategy, Responses and Legacy of the First Imperialist Era in the Sudan 1820-1885 », The Muslim World, 91 : 209-227.

ISRAEL, J. I.

2001 - Les Lumières radicales : la philosophie, Spinoza et la naissance de la modernité (1650-1750), Paris, Éditions Amsterdam.

KAPTEIJNS, L.

1985 - « Islamic Rationales for the Changing Social Roles of Women in the Western Sudan », in

M. W. DALY (ed.), op. cit. : 57-72.

KEEFE, S. E.

2008 - « Theorizing Modernity in Appalachia », Journal of Appalachian Studies, 14 (1/2) : 160-173.

LAMBERT, Y.

2000 - « Religion, modernité, ultramodernité : une analyse en terme de "tournant axial" ", Archives de sciences sociales des religions, 109, <http://assr.revues.org/index20178.html>.

LO, M.-C. M.

2002 - Doctors within Borders : Profession, Ethnicity, and Modernity in Colonial Taiwan, Berkeley, University of California Press.

Loos, $\mathrm{T}$.

2006 - Subject Siam : Family, Law, and Colonial Modernity in Thailand, Ithaca (N.Y.), Cornell University Press.

MCCLELLAND, D. C.

1961 - The Achieving Society, Princeton (N. J.), Van Nostrand.

OHTSUKA, K.

1997 - « Salafi-Orientation in Sudanese Mahdism », The Muslim World, 87 (1) : 17-33.

ORY, P.

1987 - « Les Temps modernes », in P. ORY (dir.), Nouvelle histoire des idées politiques, Paris, Hachette : 29-30.

POPPER, K.

1956 - Misère de l'historicisme, Paris, Plon.

POUCHEPADASS, J.

2000 - « Les Subaltern Studies ou la critique postcoloniale de la modernité », L'Homme, 156 :

161-185.

PRAKASH, G. (ED.)

1995 - « After Colonialism », in G. PRAKASH (ed.), After Colonialism : Imperial Histories and Postcolonial Displacements, Princeton, Princeton University Press : 3-17.

RAFFIN, A.

2008 - « Postcolonial Vietnam : Hybrid Modernity », Postcolonial Studies, 11 (3) : 329-344. 
RÉMOND, R.

1974 - Introduction à l'histoire de notre temps, 2. Le XIX'e siècle, 1815-1914, Paris, Éditions du Seuil.

RosTow, W.

1960 - The Stages of Economic Growth : A Non-Communist Manifesto, Cambridge, Cambridge

University Press.

RUFF, M.-A.

1968 - « La filiation de Baudelaire à Rimbaud », Études littéraires, 1 (1) : 67-82, <http://

www.erudit.org/revue/etudlitt/1968/v1/n1/500004ar.pdf>.

AL-SÂWî, 'A. al-‘A. H.

1994 - Hiwârât al-Hawiyya wa-l-Wahda al-Wataniyya fì al-Sûdân, Wijhat Nazar Mukhtalifa, Le Caire, Markaz al-Dirâsât al-Sûdâniyya.

SCHMIDT, V. H.

2006 - « Multiple Modernities or Varieties of Modernity? », Current Sociology, 41 (1) : 77-97.

SERI-HERSCH, I.

2012 - Histoire scolaire, impérialisme(s) et décolonisation(s) : le cas du Soudan anglo-égyptien (1945-1958), Thèse de doctorat Aix-en-Provence, Aix-Marseille Université, <http://tel.archives-ouvertes.fr/ tel-00700410>.

SHARKEY, H. J.

2003 - Living with Colonialism : Nationalism and Culture in the Anglo-Egyptian Sudan, Berkeley, University of California Press.

SHIN, G.-W. \& ROBINSON, M. (EDS.)

1999 - Colonial Modernity in Korea, Cambridge (Mass.), Harvard University Asia Center.

SMITH, A. D.

2009 - Ethno-Symbolism and Nationalism : A Cultural Approach, London, Routledge.

VAN DER VEER, P.

2001 - Imperial Encounters : Religion and Modernity in India and Britain, Princeton, Princeton University Press.

VEZZADINI, E.

2012 - « Islam and Capitalism. Considerations on the Construction of the Idea of a Western "Modernity" ", Storicamente, 8 : 1-11.

WALLERSTEIN, I.

1974, 1980, 1989 - The Modern World System, 3 vol., New York, Academic Press.

WARBURG, G. R.

1992 - Historical Discord in the Nile Valley, London, Hurst and Co.

WEBER, M.

1994 [1964] - L'éthique protestante et l'esprit du capitalisme, Paris, Plon.

WILLIS, J.

2003 - " "The Nyamang are Hard to Touch" : Mission Evangelism and Tradition in the Nuba Mountains, Sudan, 1933-1952 », Journal of Religion in Africa, 33 (1) : 32-62.

WITTROCK, B.

2000 - « Modernity : One, None, or Many ? European Origins and Modernity as a Global Condition », Daedalus, 129 (1) : 31-60. 
ZANASI, M.

2006 - Saving the Nation : Economic Modernity in Republican China, Chicago, University of Chicago

Press.

\section{NOTES}

1. Fruit d'un travail effectué dans le cadre du séminaire IREMAM «Autour de la modernité : l'Égypte et la Turquie de 1750 à nos jours ", Aix-en-Provence, 2006-2007. Je remercie les responsables du séminaire, Ghislaine Alleaume et Gérard Groc, d'avoir suscité des réflexions et des débats féconds sur la notion de modernité dans une perspective comparatiste. Dans cet article, les traductions de l'anglais et de l'arabe vers le français ont été réalisées par mes soins.

2. Parmi d'innombrables travaux, voir notamment ABU-LUGHOD (1998), ALIDOU (2005), AWAH \& PHillimore (2008), BaRTolovich et LAZARUs (2002), CHAKRABARTY $(2000,2002)$, CHAMBERS (2008), CHAUDHURI (2008), CIAFFA (2008), DE JONG (2005), FRIEDMAN (2006), HAROOTUNIAN (2001), HOLDEN (2008), LO (2002), LOOS (2006), POUCHEPADASS (2000), PRAKASH (1995), RAFFIN (2008), SHIN et ROBINSON (1999), VAN DER VEER (2001), ZANASI (2006). Les interrogations sur le couple colonialisme-modernité sont aussi une mode très actuelle dans la recherche française, voir par exemple le programme de la journée d'études «Colonisation et modernisation: un mariage improbable?» (Archives nationales d'Outre-Mer, Aix-en-Provence, 25 février 2010), <http://iremam.univ-provence.fr/IMG/ pdf/Colloque_Gantes.pdf>.

3. Voir Y. LAMBERT (2000) pour une périodisation de la modernité en trois moments : les " Temps modernes » (1492-milieu XVII ${ }^{\mathrm{e}}$ siècle), le mouvement des Lumières (milieu XVII ${ }^{\mathrm{e}}$ fin XVIII ${ }^{\mathrm{e}}$ siècle), et la société industrielle (XIX ${ }^{\mathrm{e}}$-milieu XX $\mathrm{X}^{\mathrm{e}}$ siècle).

4. Le sociologue MaX WEBER (1994) a vu dans le protestantisme une véritable révolution des esprits qui a permis l'essor du capitalisme, signe distinctif de la modernité occidentale. Transformant le rapport à l'argent et à la fortune, la Réforme luthérienne représente, selon cette perspective, un développement d'une importance capitale pour comprendre les origines du rationalisme européen.

5. Parmi les sources limitées de cette recherche, signalons ARON (1969), ELIAS (1996), GELLNER (1999) : tous trois instaurent cette quasi-synonymie à plusieurs reprises dans leurs ouvrages respectifs.

6. Pour les principales études en langue anglaise, voir ANDERSON (2002:12-13).

7. Pour les approches anglo-marxiste et ethno-symboliste du nationalisme, voir respectivement НОВSВАWм (1990) et SмIтн (2009).

8. Pour une analyse critique du lien entre capitalisme et modernité, voir GOoDY (2004).

9. Parmi les tenants de ces théories, voir Rostow (1960) et mCCLELland (1961). Pour une critique de ces théories, voir par exemple HABERMAS (1988).

10. Au terme « turco-égyptien " généralement utilisé par les historiens pour désigner le régime que l'Égypte ottomane instaura au Soudan au XIX ${ }^{e}$ siècle, je préfère l'expression

"ottomano-égyptien», historiquement plus précise. L'expression «histoire contemporaine » est ici employée à la manière conventionnelle de l'historiographie française (XIX et $\mathrm{XX}^{\mathrm{e}}$ siècles) et comme traduction de l'anglais « modern history ». 
11. Le style architectural concerne très peu de bâtiments : ceux de la mission catholique romaine et le palais du gouverneur général ottomano-égyptien.

12. Robert O. collins (1967: 79-80) reprend quasiment les idées de Hill en présentant l'héritage de la Turkiyya (le règne ottomano-égyptien) dans un chapitre intitulé «The Beginnings of Modernization». Il insiste toutefois plus sur l'unification politique du Soudan et fait remonter les origines de la guerre civile entre le Nord et le Sud-Soudan à la période de la Turkiyya. Son ouvrage récent (Collins 2008: 10-20) ne dévie pas de cette ligne.

13. Il s'agit là de la préface à la première édition (1961), conservée dans la cinquième édition (2000).

14. Cet aspect politique et administratif est également souligné par un historien soudanais dans une thèse de doctorat soutenue à l'université de Cambridge en 1965. Voir BАКнÎT (1987 : 21) (traduction arabe de cette thèse).

15. «Elle-même un produit du mélange entre Musulmans arabes et Africains » (HoLt \& DALY 2000: VIII). Notons la dichotomie réductrice entre une présumée "tradition » autochtone et des « influences » extérieures.

16. Voir COOPER (2005: 130) pour une critique similaire du concept de la modernité.

17. Notamment Samuel W. Baker (1869-1873), Charles G. Gordon (1874-1880) et Romolo Gessi (1874-1880).

18. «[...] au Soudan les Britanniques conçurent le Gordon College comme une machine à effendis destinée à produire des hommes modernes connaissant suffisamment le style et les objectifs britanniques pour pouvoir travailler au sein d'une administration anglaise. » Qui qualifie ces hommes de "modernes", Sharkey ou les responsables du Gordon College?

19. «En tant que symbole majeur de la modernité aux yeux du colonisé, les vêtements occidentaux marquaient le progrès et l'accomplissement professionnels. " Sharkey soutient cette généralité en citant HENDRICKSON (1996) plutôt qu'une source primaire précise.

20. Pour les théories marxistes de la dépendance et la conception d'un système-monde capitaliste, voir respectivement FRANK (1974) et WALLERSTEIN $(1974,1980,1989)$.

21. D'une manière assez intéressante, AL-GADDÂL (1985:53) lie « l'intensification de la lutte des classes dans la société musulmane » à la survivance de l'idée du mahdî (figure eschatologique de l'islam sunnite supposée restaurer la vraie religion et la justice) dans cette société. Plus loin, il dépeint l'État mahdiste [État islamique indépendant (1885-1898) établi suite à la révolution qui renversa le régime ottomano-égyptien au Soudan] comme l'instrument de la «classe marchande » issue essentiellement des tribus riveraines du Soudan central (ibid. : 55).

22. AL-GADDÂL (ibid.: 16) insiste toutefois sur l'impossibilité d'affirmer que l'année 1821 est le commencement de l'histoire contemporaine du Soudan. En effet, selon lui, les changements caractéristiques de la modernité ne se produisent ni soudainement, ni en l'espace de quelques années seulement.

23. Les grands éléments de la civilisation moderne tels qu'ils furent suggérés par l'écrivain et historien écossais Thomas CARLYLE (2010 : 28) en 1827. 
24. Les trois principales missions actives au Sud-Soudan dès le début du Condominium anglo-égyptien (1899-1956) étaient la Church Missionary Society, les Verona Fathers et l'American Presbyterian Mission, voir BURTON (1985 : 353).

25. Il s'agissait d'administrer séparément le Sud et le Nord du pays en limitant le plus possible les contacts entre les deux régions. Cette politique fut officiellement abandonnée à la Conférence de Juba en 1947.

26. La nudité forcée outragea les filles de l'école qui contraignirent la directrice à changer de politique.

27. Kapteijns fait un usage essentialiste et extrêmement vague de la notion de Nile Valley ideology.

28. L'auteur s'appuie sur la distinction proposée par E. GELLNER (1969) entre deux types de religiosité en islam, l'une scripturaire et égalitaire, l'autre ritualisée et hiérarchisée. Gellner désigne ces deux tendances de l'islam par les lettres $P$ et $C$ pour suggérer un parallèle avec le protestantisme et le catholicisme européens.

29. En septembre 1983, le président Gaafar Nimeiry décida subitement d'instaurer de nouvelles lois inspirées de la sharîa islamique.

30. Autre terme employé par COOPER $(2005: 6,11)$ pour désigner les catégories normatives.

31. Voir par exemple APPADURAi (1996), CHAKRABARTY $(2000,2002)$, EISENSTADT (2000), FRIEDMAN (2006), GAONKAR (2001), KEEFE (2008), LOOS (2006), VEZZADINI (2012), WITTRoCK (2000). Des postures critiques à l'encontre de la démultiplication de la modernité ont été adoptées par COOPER $(2005: 114,133)$ et SCHMIDT $(2006)$.

32. Sur la modernité en tant que catégorie mobilisée par les pédagogues coloniaux du Soudan dans les années 1940 et 1950 voir SERI-HERSCH (2012 : 129-130, 223-225).

\section{RÉSUMÉS}

Résumé

Cet article propose une réflexion critique sur les usages de la catégorie "modernité » dans l'historiographie du Soudan contemporain. Attentive à la distinction heuristique entre catégories analytiques et catégories normatives, l'étude revient sur des conceptualisations européennes de la modernité avant d'examiner ses usages dans la production académique soudaniste des cinquante dernières années. En dépit de la diversité de leurs approches et de leurs objets, la plupart des travaux analysés endossent explicitement ou implicitement l'une ou l'autre des théories de la modernisation et de la dépendance. L'argumentaire débouche sur la nécessité, pour les chercheurs en sciences sociales, de sortir des impasses épistémologiques de la modernité « modernisatrice » et des modernités « multiples».

Scrutinizing modernity through the historiography of Sudan: conventional uses of a nebulous concept?

This paper deals with "modernity" as an analytical category, investigating how it has been used by scholars of modern Sudan in the last fifty years. Keeping in mind the heuristic distinction 
between analytical and normative categories, the study goes back to European conceptualizations of modernity before examining how it has been constructed and used across a wide range of Sudanist academic writings. Despite significant differences in their approaches and subjects of study, most of the works under review explicitly or implicitly adopt either modernization or dependence theories. The paper ultimately argues for the necessity of avoiding the epistemological dead ends of both "modernizing" and "multiple" modernities.

INDEX

Mots-clés : Soudan, épistémologie, études postcoloniales, historiographie, modernisation, modernité

Keywords : Sudan, Epistemology, Postcolonial Studies, Historiography, Modernization, Modernity

\section{AUTEUR}

\section{IRIS SERI-HERSCH}

Institut de Recherches et d'Études sur le Monde Arabe et Musulman, Aix-en Provence. 